\title{
On Power/Bandwidth Efficient Clipped-OFDM and SC/FDE Transmission Schemes with Reduced CP Overhead
}

\author{
Paulo Torres \\ IPCB-EST, Polyt. Inst. Castelo Branco, Portugal, \\ Email: paulo.torres@ieee.org
}

\author{
António Gusmão \\ CAPS-IST, Tech. Univ. of Lisbon, Portugal, \\ Email: gus@ist.utl.pt
}

\begin{abstract}
In this paper, we consider two reduced-PMEPR (Peak to Mean Envelope Power Ratio), Reduced-CP (Cyclic Prefix) block transmission alternatives, having in mind an application to the uplink of future mobile broadband systems: clipped (and filtered) OFDM (Orthogonal Frequency Division Multiplexing) and SC/FDE (Single Carrier/Frequency-Domain Equalization). In both cases, we adopt advanced receivers with space diversity and a similar structure, where an iterative cancellation of unavoidable interferences is carried out: interference due to a $\mathrm{CP}$ length below the channel memory order, in both cases; deliberate nonlinear interference, in the Clipped-OFDM case; residual linear ISI, in the SC/FDE case. Performance results, when a 16-QAM (Quadrature Amplitude Modulation) constellation is adopted in both cases, are reported and discussed, with the help of selected performance bounds. These results show that we can add the benefits of a reduced $\mathrm{CP}$ to those of a reduced PMEPR, through the use of the proposed iterative receiver techniques, thereby providing a highly power/bandwidth efficient block transmission, with both Clipped-OFDM and SC/FDE schemes. The proposed OFDM receiver technique is shown to ensure the feasibility of a clipping effort strong enough to reduce the OFDM PMEPR to a level below that of the corresponding SC/FDE scheme; however, it is not able to prevent an overall advantage for the SC/FDE side, namely under a strongly reduced $\mathrm{CP}$ overhead.
\end{abstract}

\section{INTRODUCTION}

A CP-assisted block transmission is widely accepted as a good choice for future mobile systems, taking advantage of current low-cost, flexible, FFT-based (Fast Fourier Transform) signal processing technology with both OFDM and SC/FDE alternatives [1], [2], [3]. OFDM schemes suffer from strong envelope fluctuations, requiring quasi-linear power amplification and significant power backoffs. One approach to reduce the PMEPR at the amplifier input involves a deliberate nonlinear distortion [4]; then, an iterative cancellation of that distortion can be implemented at the receiver, as shown in [5], [6]. On the other hand, conventional SC modulations with a reduced PMEPR are easily designed; under a full-length $\mathrm{CP}$, a low-complexity FDE technique can be employed to solve the severe ISI problem. The possibility of achieving improved FDE performances in SC-based systems was demonstrated in [7]. Recently, an alternative Turbo FDE approach, which is able to deal with reduced-CP conditions, was developed [8]. This "reduced-CP approach" was later extended to the Clipped-OFDM case [9].

In a recent paper [10], we considered a reducedPMEPR, Clipped-OFDM transmission, with an advanced receiver performing the iterative cancellation of the deliberate nonlinear distortion effects. In parallel, we considered an advanced Turbo FDE receiver within the SC/FDE alternative. In both cases, a 4-QAM constellation (as in [8] and [9]) and a full-length CP have been assumed. The "SC vs OFDM" comparisons of that paper, under the use of iterative receiver techniques in both cases, have shown a very significant overall advantage for the SC/FDE side.

In this paper, we consider again the two reducedPMEPR block transmission alternatives which have been preliminarily compared in [10], in the context of an iterative receiver implementation. Having in mind applications to the uplink of future broadband systems, a receiver implementation with space diversity is considered here. For the sake of increased bandwidth efficiency, within a cellular system, we also consider a CP length below the channel memory order and a 16-QAM constellation, for both Clipped-OFDM and SC/FDE. A 16-state, rate $1 / 2$ convolutional coding is assumed, under a BICM (Bit Interleaved Coded Modulation) approach [11].

Sec. II defines a Q-branch receiver structure to be adopted here for iterative, joint cancellation of the unavoidable interferences: interference due to a $\mathrm{CP}$ length below the channel memory order, in both cases; deliberate nonlinear interference, in the Clipped-OFDM case; residual linear ISI, in the SC/FDE case. Sec. III and IV deal with the specific techniques to be implemented within that receiver structure, for SC/FDE and Clipped-OFDM, respectively. Performance results are reported and discussed in sec. V, with the help of selected performance bounds, thereby providing relevant "SC vs OFDM" comparisons and leading to the overall conclusions.

\section{REDUCED-PMEPR, REDUCED-CP BLOCK TRANSMISSION}

It should be noted that the two transmission alternatives considered in this paper correspond to two different ways of implementing a PMEPR-reducing SP (Signal Processing) block within an "OFDM-type" transmitter structure (see Fig. 1): a nonlinear SP, for Clipped-OFDM transmission; a linear SP, according to a DFT matrix, for SC/FDE transmission (due to the cancellation effect which is inherent to the resulting DFT/IDFT pair) [12].

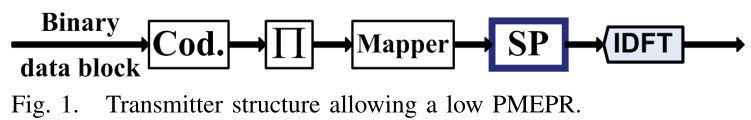




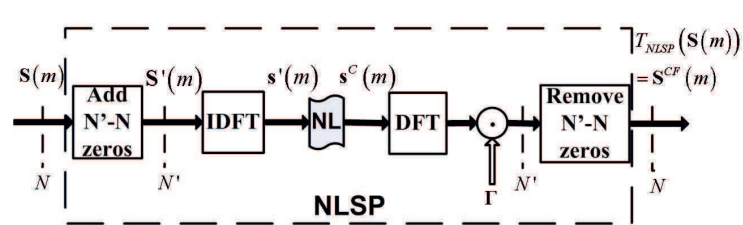

Fig. 2. Nonlinear signal processing scheme (NLSP) to reduce the PMEPR of OFDM signals, while preserving the spectral efficiency of ordinary OFDM.

Regarding the nonlinear SP option, we adopt here the signal processing scheme described in detail in [4]. The modified blocks $\mathbf{S}^{C F}(m)=\left[S_{0}^{C F}(m), \cdots, S_{N-1}^{C F}(m)\right]^{T}$ are obtained from the conventional blocks $\mathbf{S}(m)=$ $\left[S_{0}(m), \cdots, S_{N-1}(m)\right]^{T}$ as shown in Fig. 2, through a nonlinear (NL), time-domain clipping operation, followed by a frequency-domain filtering. It is assumed here a clipping level $s_{M}, N^{\prime} / N=2$ and a complementary frequency-domain filtering characterized by $\Gamma_{k}=0$ or 1 ("out-of-band" or "in-band", respectively). For $N>>1$, both $\Re e\left\{s_{n}^{\prime}(m)\right\}$ and $\Im m\left\{s_{n}^{\prime}(m)\right\}$ are quasi-Gaussian, with zero mean and a given variance $\sigma^{2}$. The transmitted frequency-domain samples can be decomposed into two uncorrelated terms, as follows [4]:

$$
S_{k}^{C F}(m)=\beta S_{k}(m)+D_{k}(m),
$$

where $\beta=1-\exp \left(-k_{M}^{2} / 2\right)+\frac{1}{2} \sqrt{2 \pi} k_{M} Q\left(k_{M}\right)$, for $k_{M}=s_{M} / \sigma$, and $D_{k}(m)$ exhibits quasi-Gaussian characteristics for any $k$ under a "high $N$ " assumption $\left(Q\left(k_{M}\right)=\frac{1}{\sqrt{2 \pi}} \int_{k_{M}}^{+\infty} \exp \left(-x^{2} / 2\right) d x\right)$.

When employing the nonlinear processing scheme described above, a certain performance degradation is unavoidable with a conventional OFDM receiver: rather small for reduced clipping efforts $\left(s_{M} / \sigma>3.5\right)$, but significant when very low PMEPR values are intended $\left(s_{M} / \sigma<1.5\right)$. In the conventional single-branch OFDM receiver, after guard removal, each block of received samples $\mathbf{y}(m)$ is converted into a frequency-domain block $\mathbf{Y}(m)=\left[Y_{0}(m), \cdots, Y_{N-1}(m)\right]^{T}$ by a DFT processor. $Y_{k}(m)=S_{k}^{C F}(m) H_{k}+N_{k}(m)$, where $N_{k}(m)$ and $H_{k}$ concern the Gaussian noise and the overall channel response, respectively, at the $k$ th sub-channel, and $S_{k}^{C F}(m)$ is given by (1). The performance degradation with the deliberate nonlinear distortion is jointly due to the fact that a "useless" power is spent as a transmitted selfinterference (see (1)) and to the fact that the received quasi-Gaussian self-interference on each subcarrier is added to the Gaussian channel noise.

The receiver structure proposed here for both SC/FDE and Clipped-OFDM is depicted in Fig. 3, with appropriately defined vectors, $\mathbf{C}^{(i)}(m)$ and $\mathbf{G}^{(i)}(m)$ (for the $m^{t h}$ block, with $i$ as the iteration number), as explained in Sec. III (SC/FDE) and Sec. IV (Clipped-OFDM). $\mathbf{G}^{(i)}(m)$ is used for iterative interference cancellation purposes, in both cases; this vector and $\mathbf{C}^{(i)}(m)$ are generated by block R (a soft "remapping" is involved), taking into account the SISO (Soft-In, Soft-Out) decoder outputs. $\Pi$ and $\Pi^{-1}$ stand for "interleaver" and "deinterleaver",

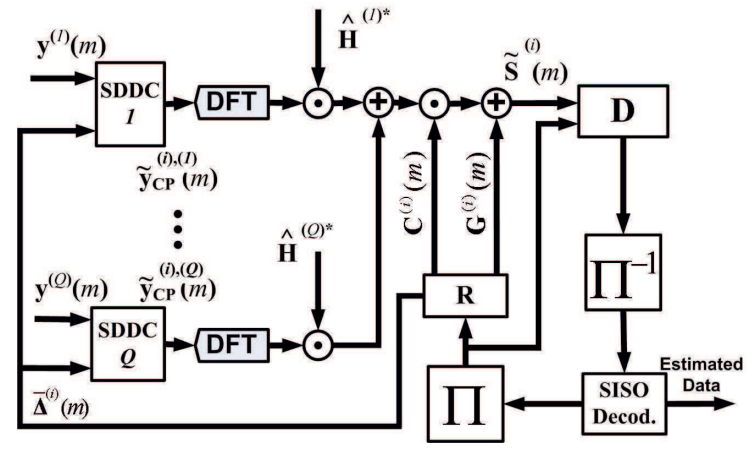

Fig. 3. Receiver structure using interference cancellation techniques.

respectively, and $\odot$ denotes "element-by-element multiplication". Block D concerns the required soft demapping (after an additional IDFT, obviously, in the SC/FDE case); the SISO decoder outputs can be used as a demapping aid (see Appendix). Block $\mathbf{R}$ also generates the vector $\bar{\Delta}^{(i)}(m)$ required for an appropriate "SDDC operation" (Soft Decision-Directed Correction) [8], [9] on each receiver branch, so as to deal with the reduced $\mathrm{CP}$ assumed here (CP length $L_{R}$ below the channel memory order $L$ ). This vector is given by

$$
\begin{array}{r}
\overline{\boldsymbol{\Delta}}^{(i)}(m)= \\
{[\underbrace{0 \ldots 0}_{N-L}, \underbrace{\overline{\Delta s}_{N-L}^{(i)}(m) \ldots \overline{\Delta s}_{N-L_{R}-1}^{(i)}(m)}_{\Delta L}, \underbrace{0 \ldots 0}_{L_{R}}]^{T},}
\end{array}
$$

where

$$
\overline{\Delta s}_{p}^{(i)}(m)=\bar{s}_{p}^{(i-1)}(m)-\bar{s}_{p+L_{R}}^{(I)}(m-1)
$$

in the SC/FDE case, for $p=N-L, \cdots, N-L_{R}-1$, with $\bar{s}_{p}^{(i-1)}(m)$ (preceding iteration for the current block) and $\bar{s}_{p+L_{R}}^{(I)}(m-1)$ (last iteration for the preceding block) being mean symbol values, derived from the SISO decoder outputs. With Clipped-OFDM, (3) should be replaced by ${\overline{\Delta s_{p}^{C F}}}^{(i)}(m)={\overline{s_{p}^{C F}}}^{(i-1)}(m)-{\overline{s_{p+L_{R}}^{C F}}}^{(I)}(m-1)$, since the time-domain symbol coefficients are derived from a modified version (according to $\left.T_{N L S P}().\right)$ of the estimated frequency-domain symbol coefficients.

\section{ITERATIVE RECEIVER TECHNIQUE FOR THE REDUCED-CP SC/FDE CASE}

Within the Q-branch receiver structure of Fig. 3, when used for SC/FDE CP-assisted transmission with length-N symbol blocks, $\mathbf{G}^{(i)}(m)$ and $\mathbf{C}^{(i)}(m)$ can be computed as explained below, following an extension of the approach adopted in [8] and [10]. The required time-domain equalizer output $\widetilde{\mathbf{s}}^{(i)}(m)$ is the IDFT of

$\tilde{\mathbf{S}}^{(i)}(m)=\sum_{q=1}^{Q} \mathbf{F}^{(i),(q)}(m) \odot \tilde{\mathbf{Y}}_{C P}^{(i),(q)}(m)+\mathbf{G}^{(i)}(m)$,

where $\mathbf{F}^{(i),(q)}(m)=\widehat{\mathbf{H}}^{(q) *} \odot \mathbf{C}^{(i)}(m)$ (when vectors $\mathbf{H}^{(q)}$ are used to denote the $Q$ channel frequency responses). A "soft demapping" procedure based on the $I$ and $Q$ 
components of $\widetilde{\mathbf{s}}^{(i)}(\mathrm{m})$ provides the inputs to the SISO decoder (LLRs of the coded bits, obtained as shown in [8], [10] and Appendix). The components of $\mathbf{G}^{(i)}(m)$, for iteration $i>1$, are given by

$$
\begin{aligned}
G_{k}^{(i)}(m) & =\left(\gamma^{(i)}(m)-\sum_{q=1}^{Q} F_{k}^{(i),(q)}(m) \hat{H}_{k}^{(q)}\right) \times \\
& \times \bar{S}_{k}^{(i-1)}(m)
\end{aligned}
$$

(for $i=1, G_{k}^{(i)}(m)=0$ ), where

$$
\begin{aligned}
\gamma^{(i)}(m) & =\frac{1}{N} \sum_{k=0}^{N-1} \sum_{q=1}^{Q} F_{k}^{(i),(q)}(m) \hat{H}_{k}^{(q)} . \\
\overline{\mathbf{S}}^{(i-1)}(m) & =\left[\bar{S}_{0}^{(i-1)}(m), \cdots, \bar{S}_{N-1}^{(i-1)}(m)\right]^{T} \text { is the }
\end{aligned}
$$
DFT of the "average symbol vector" currently available, $\overline{\mathbf{s}}^{(i-1)}(m)=\left[\bar{s}_{0}^{(i-1)}(m), \cdots, \bar{s}_{N-1}^{(i-1)}(m)\right]^{T}$, which can be easily derived from the SISO decoder outputs in a "soft remapping" procedure (see [8], [10] and Appendix); these outputs must correspond to the full soft information on the coded bits, not the extrinsic one. As to $\mathbf{C}^{(i)}(m)$, the approach of [8], [10] leads to components

$$
C_{k}^{(i)}(m)=\frac{K_{F}^{(i)}(m)}{\hat{\alpha}+\left[1-\left(\hat{\rho}^{(i-1)}(m)\right)^{2}\right] \sum_{q=1}^{Q}\left|\hat{H}_{k}^{(q)}\right|^{2}},
$$

where $\alpha=\sigma_{n}^{2} / \sigma_{s}^{2}\left(\sigma_{n}^{2}\right.$ denoting the variance of the timedomain noise components of the $Q$ receiver inputs, and $\sigma_{s}^{2}=\overline{\left|s_{n}\right|^{2}}$ denoting the variance of the time-domain data symbols). $K_{F}^{(i)}(m)$ is a normalization factor (e.g, so that $\gamma^{(i)}(m)=1$ in $\left.(5)\right) . \hat{\rho}^{(i-1)}(m)$ is an overall measure of the reliability of possible coded bit decisions derived from the decoder outputs currently available: as explained in [8] and [10] (see also in Appendix), $\hat{\rho}^{(i-1)}(m)$ can be obtained as an average value of the several "correlation coefficients" per bit, each one between 0 and 1. For $i=1$ (with no decoder outputs available), the $F_{k}^{(i),(q)}(m)$ parameters meet the MMSE criterion, since $\hat{\rho}^{(i-1)}(m)=0$. After a number of iterations and/or high SNRs, typically $\hat{\rho}^{(i-1)}(m) \approx 1$, leading to $F_{k}^{(i),(q)}(m)$ approximately in accordance with the MF criterion.

\section{ITERATIVE RECEIVER TECHNIQUE FOR THE REDUCED-CP CLIPPED-OFDM CASE}

Within the $Q$-branch receiver structure of Fig. 3, when used for Clipped-OFDM block transmission with length$\mathrm{N}$ symbol blocks, the vectors $\mathbf{G}^{(i)}(m)$ and $\mathbf{C}^{(i)}(m)$ can be computed as explained below, by extending the approach adopted in [10]. With Clipped-OFDM,

$$
C_{k}^{(i)}(m)=\left[\beta \sum_{q=1}^{Q}\left|\hat{H}_{k}^{(q)}\right|^{2}\right]^{-1} .
$$

On the other hand, as explained in [10], for $i>1$,

$$
\begin{aligned}
\mathbf{G}^{(i)}(m) & =\hat{\rho}^{(i-1)}(m) \times \\
& \times\left[\hat{\mathbf{S}}^{(i-1)}(m)-\frac{1}{\beta} T_{N L S P}\left(\hat{\mathbf{S}}^{(i-1)}(m)\right)\right]
\end{aligned}
$$

$\left(G^{(1)}(m)=0\right)$, where $\hat{\rho}^{(i-1)}(m)$ is the overall reliability factor derived from the LLRs of all coded bits, provided by the SISO decoder at the preceding iteration, and the components of $\hat{\mathbf{S}}^{(i-1)}(m)$ are $\hat{S}_{k}^{(i-1)}(m)=\hat{S}_{k, I}^{(i-1)}(m)+$ $j \hat{S}_{k, Q}^{(i-1)}(m)$, with $\hat{S}_{k, I}^{(i-1)}(m)$ and $\hat{S}_{k, Q}^{(i-1)}(m)$ as "inphase" and "quadrature" symbol decisions based on the same SISO decoder outputs (see Appendix). By adding $\mathbf{G}^{(i)}(m)$ to obtain $\widetilde{\mathbf{S}}^{(i)}(m)$, we are simply subtracting an estimate of the nonlinear distortion. Due to the nonlinear nature of the $T_{N L S P}($.$) operation, it is not appropriate$ to use $\overline{\mathbf{S}}^{(i-1)}(m)$ directly when computing $\mathbf{G}^{(i)}(m)$, and the use of (8) is realistic for creating the intended soft cancellation effect.

As to the SDDC operations (reported in Sec. II), for cancellation of the interference resulting from the reduced $\mathrm{CP}$, the required $\overline{\mathbf{S}^{\mathbf{C F}}}{ }^{(i-1)}(m)$ vector is the IDFT of a frequency-domain vector $\overline{\mathbf{S}}^{(i-1)}(m)$ given by $\widehat{\rho}^{(i-1)}(m) \cdot T_{N L S P}\left(\widehat{\mathbf{s}}^{(i-1)}(m)\right)$ (and similarly for $\left.\overline{\mathbf{S}}^{\mathbf{C F}}(I)(m-1)\right)$.

\section{PeRformance Comparisons AND CONCLUSIONS}

In the following, we present a set of numerical performance results, with regards to broadband transmission over a strongly frequency-selective Rayleigh fading channel, when the iterative receiver techniques of Secs. III and IV are employed (power delay profile type C within HIPERLAN/2). A CP-assisted block transmission is assumed, with $N=256$ and a 16-QAM constellation for both SC/FDE and Clipped-OFDM $\left(k_{M}=0.5\right)$ schemes. In both cases, we selected a 16-state, rate-1/2 convolutional code with $G(D)=\left[\begin{array}{ll}1 & \frac{1+D^{2}+D^{3}+D^{4}}{1+D+D^{4}}\end{array}\right]$ and a low-complexity Max-Log-MAP SISO decoding [13]. The duration of the useful part of each block is $5 \mu \mathrm{s}$, and either a full-length $\mathrm{CP}\left(L_{R}=L=64\right)$ or a reduced CP $\left(L_{R}=L / 8=8\right)$ are adopted.

Fig. 4 is concerned to performance results with fulllength CP (the "SDDC aid" is not relevant here, since $\left.\overline{\boldsymbol{\Delta}}^{(i)}(m)=\mathbf{0}\right)$ for $Q=1(a)$ and $Q=2(b)$. We include lower bounds on the achievable $B E R$, which have been obtained by simulation, when assuming perfect interference cancellation through an ideal $\mathbf{G}^{(i)}(m)$, and also an ideal $\mathbf{C}^{(i)}(m)$ (through $\widehat{\rho}^{(i-1)}(m)=1$ in (6)) in the SC/FDE case, leading to a true "matched filter bound". Five iterations are shown to be enough for a good approximation to each lower bound, especially in the $\mathrm{SC} / \mathrm{FDE}$ case and/or when $Q=2$ (differences below $1 \mathrm{~dB}$ at $B E R=10^{-5}$ ). Also having in mind the results of [10], concerning $4-Q A M$ constellations (and no diversity), and the several PMEPR values $\{4.1 d B$, for ClippedOFDM $\left(k_{M}=0.5\right) ; 2.8 d B$ and $4.7 d B$, respectively, for $S C / 4-Q A M$ and $S C / 16-Q A M\}$, we can conclude that the $S C / F D E$ power efficiency advantage decreases when the constellation size is increased, namely when using receiver diversity: this is due to the fact that, contrarily to the $4-Q A M$ case, the same clipping effort $\left(k_{M}=0.5\right)$ with $O F D M / 16-Q A M$ is strong enough 

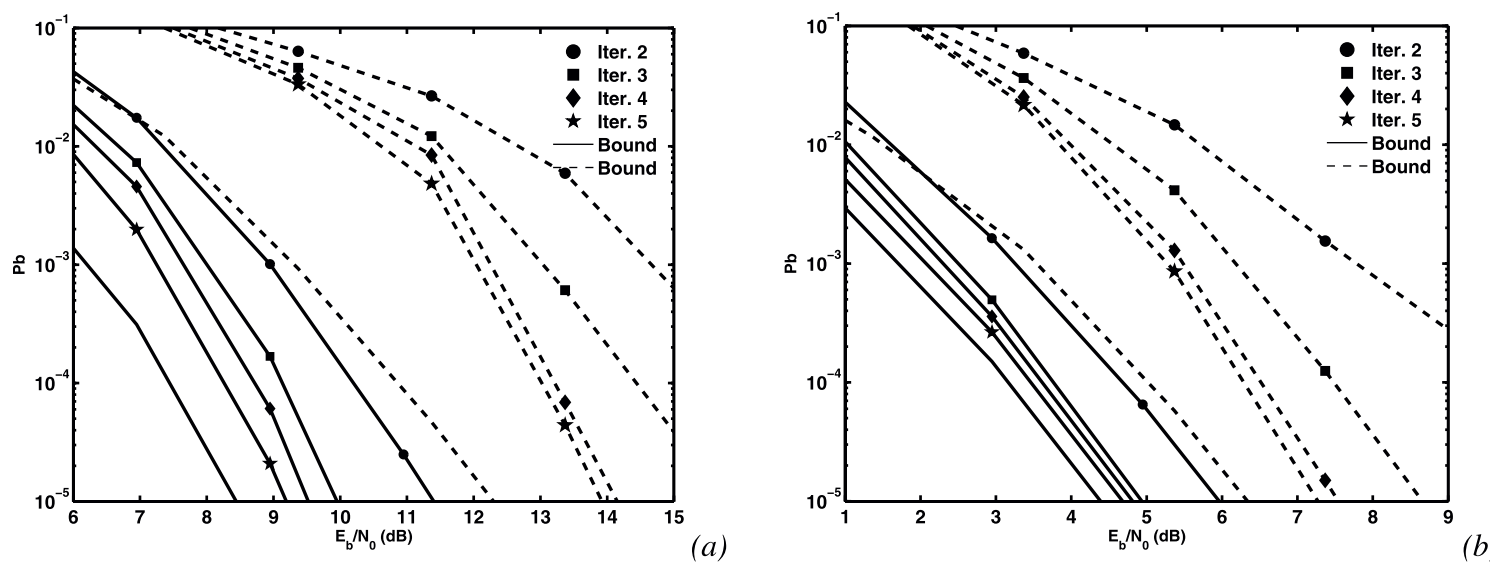

Fig. 4. SC/FDE (solid lines) vs Clipped-OFDM (dashed lines) performance comparisons, for $Q=1(a)$ and $Q=2(b)$, when $L_{R}=L=64$.
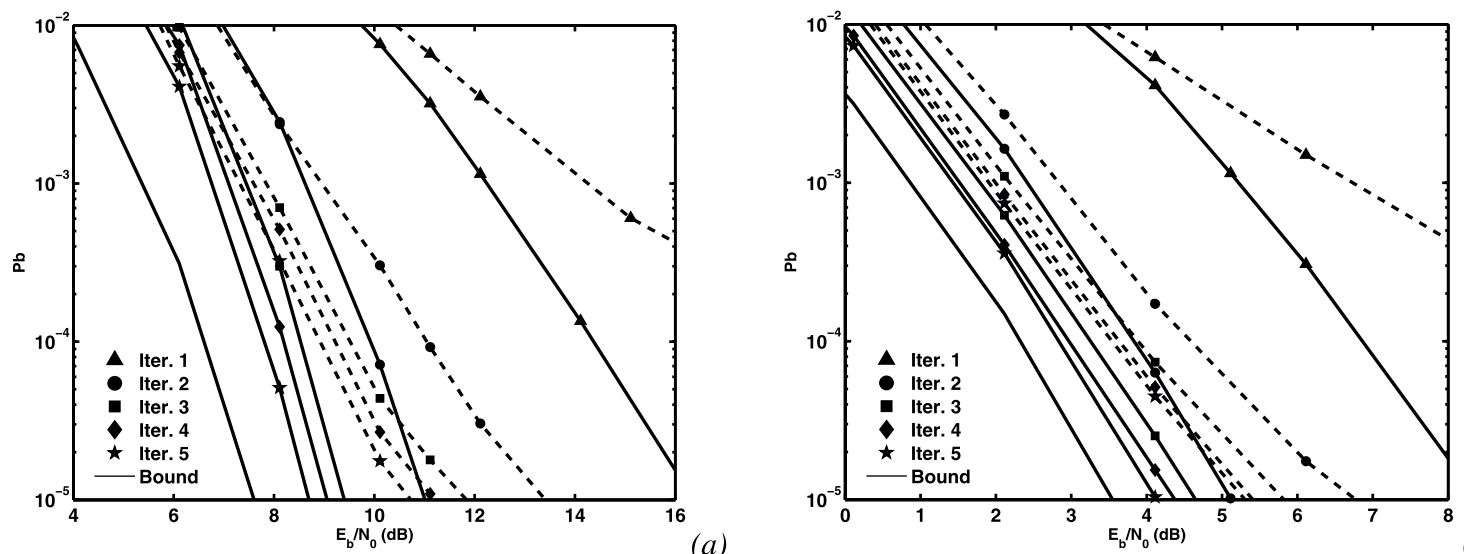

(a)

Fig. 5. Performance comparisons regarding SC/FDE with (solid lines) or without (dashed lines) "SDDC aid", for $Q=1(a)$ and $Q=2(b)$, when $L_{R}=L / 8=8$.
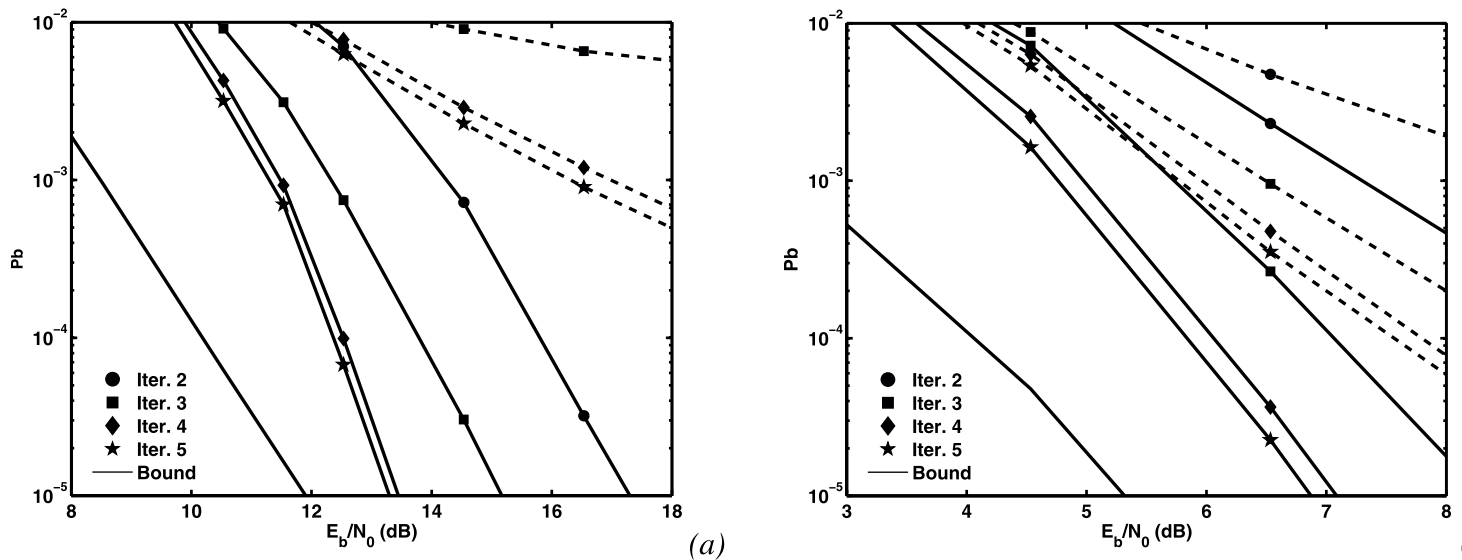

(a)

Fig. 6. Performance comparisons regarding Clipped-OFDM with (solid lines) or without (dashed lines) "SDDC aid", for $Q=1(a)$ and $Q=2(b)$, when $L_{R}=L / 8=8$.

to reduce the OFDM PMEPR to a level below that of the corresponding SC/FDE scheme.

A set of numerical results on BER performance with reduced-CP SC/FDE transmission and Clipped-OFDM transmission are depicted in Figs. 5 and 6, respectively, for $Q=1(a)$ and $Q=2(b)$; the receiver techniques of secs. III (SC/FDE) and IV (Clipped-OFDM) have been assumed, when using the receiver structure of Fig. 3 (which provides an "SDDC aid" to cope with the reduced $\mathrm{CP}$ ) or a simplified structure which does not 
provide the "SDDC aid". As for Fig. 4, we include simulated lower bounds under the same assumptions, and also under the additional assumption of an ideal SDDC operation. The "SDDC aid" is shown to be relevant for a good performance, especially in the Clipped-OFDM case, allowing reasonable approximations to the lower bound with five iterations only (differences below $1 d B$ and $1.5 \mathrm{~dB}$, respectively, for SC/FDE and Clipped-OFDM, at $B E R=10^{-5}$ ).

From these results, we can conclude that, through the use of the proposed iterative receiver techniques, a power/bandwidth efficient block transmission can be achieved, with both Clipped-OFDM and SC/FDE. The proposed OFDM receiver technique ensures the feasibility of a clipping effort strong enough to reduce the OFDM PMEPR to a level below that of the corresponding $\mathrm{SC} / \mathrm{FDE}$ scheme; however, it is not able to prevent an overall advantage for the SC/FDE side, namely when using a strongly reduced $\mathrm{CP}$ overhead.

\section{APPENDIX}

For $L-Q A M$ constellations $\left(L=2^{2 l}\right)$, in general, we can assume a separate Gray mapping with both the real and the imaginary part of the symbol coefficients ( $l$ bits for each one). In the following, we consider 16-QAM constellations, i.e., $l=2$ with both "inphase" and "quadrature" symbol components (extensions to larger QAM constellations are straightforward), within a SC-based block transmission: therefore, $\Re e\left\{s_{n}\right\}=$ $s_{n, I}=s\left(c_{n, I}, c_{n, I}^{\prime}\right)=3 \frac{\sigma_{s}}{\sqrt{10}}, \frac{\sigma_{s}}{\sqrt{10}},-\frac{\sigma_{s}}{\sqrt{10}},-3 \frac{\sigma_{s}}{\sqrt{10}}$, for $\left(c_{n, I}, c_{n, I}^{\prime}\right)=(1,1),(1,-1),(-1,-1)$ or $(-1,1)$, respectively (and similarly for $\Im m\left\{s_{n}\right\}=s_{n, Q}=$ $s\left(c_{n, Q}, c_{n, Q}^{\prime}\right)$, regarding the "quadrature" coded dibit). Consequently, one can assume $\overline{s_{n}}=0$ and $\overline{\left|s_{n}\right|^{2}}=\sigma_{s}^{2}$ (a priori values).

Since $s_{n, I}=\frac{\sigma_{s}}{\sqrt{10}}\left(2 c_{n, I}+c_{n, I} c_{n, I}^{\prime}\right)$, the remapping procedure can be implemented as follows, using the LLRs $\left(L_{n, I}, L_{n, I}^{\prime}, L_{n, Q}, L_{n, Q}^{\prime}\right)$ of the coded bits $c_{n, I}, c_{n, I}^{\prime}, c_{n, Q}, c_{n, Q}^{\prime}$, provided by the SISO decoder in the preceding iteration: $\bar{s}_{n, I}=\frac{\sigma_{s}}{\sqrt{10}}\left(2 \bar{c}_{n, I}+\bar{c}_{n, I} \bar{c}^{\prime}{ }_{n, I}\right)$, where $\bar{c}_{n, I}=\tanh \left(L_{n, I} / 2\right)$ and ${\overline{c^{\prime}}}_{n, I}=\tanh \left(L_{n, I}^{\prime} / 2\right)$ (and similarly for $\bar{s}_{n, Q}$, using $L_{n, Q}$ and $L_{n, Q}^{\prime}$ ). As in [8] and [10], we define "correlation" coefficients per bit $\left(\rho_{n, I}, \rho_{n, I}^{\prime}, \rho_{n, Q}, \rho_{n, Q}^{\prime}\right)$ and virtual bit decisions $\left(\widehat{c}_{n, I}, \widehat{c}_{n, I}^{\prime}, \widehat{c}_{n, Q}, \widehat{c}_{n, Q}^{\prime}\right)$ using the LLRs of the coded bits: $\rho_{n, I}=\tanh \left(\left|L_{n, I}\right| / 2\right)=\left|\bar{c}_{n, I}\right|$ and $\widehat{c}_{n, I}=\operatorname{sgn}\left(L_{n, I}\right)=$ $\operatorname{sgn}\left(\bar{c}_{n, \mathrm{I}}\right)$, etc.

Using the well known $\max ^{*}($.$) function [13], an appro-$ priate demapping can be derived from $\widetilde{s}_{n, I}^{(i)}=\Re e\left\{\widetilde{s}_{n}^{(i)}\right\}$ (and similarly for the "quadrature" component), also taking into account the decoder outputs from the preceding iteration:

$$
\begin{array}{r}
L_{n, I}^{(i)}=\sum_{p=0}^{1}(-1)^{p} . \\
\max _{\substack{c=(-1)^{p} \\
c^{\prime}=-1,+1}}{ }^{*}\left[\frac{c^{\prime}+1}{2} L_{n, I}^{\prime(i-1)}-\frac{1}{\hat{\sigma}_{e q}^{2}}\left(\tilde{s}_{n, I}^{(i)}-s\left(c, c^{\prime}\right)\right)^{2}\right]
\end{array}
$$

and

$$
\begin{array}{r}
L_{n, I}^{\prime(i)}=\sum_{p=0}^{1}(-1)^{p} . \\
\max _{\substack{c^{\prime}=(-1)^{p} \\
c=-1,+1}}^{*}\left[\frac{c+1}{2} L_{n, I}^{(i-1)}-\frac{1}{\hat{\sigma}_{e q}^{2}}\left(\tilde{s}_{n, I}^{(i)}-s\left(c, c^{\prime}\right)\right)^{2}\right]
\end{array}
$$

where $\widehat{\sigma}_{e q}^{2}$ denotes the estimated mean-squared error in the $\widetilde{s}_{n}^{(i)}$ samples [8], [10].

For 16QAM/OFDM, the required computations are very similar, with $\sigma_{S}^{2}=\overline{\left|S_{k}\right|^{2}}, \widehat{S}_{k, I}=\frac{\sigma_{S}}{\sqrt{10}}\left(2 \widehat{c}_{k, I}+\right.$ $\left.\widehat{c}_{k, I} \widehat{c}_{k, I}\right)$ where $\widehat{c}_{k, I}=\operatorname{sgn}\left(L_{\mathrm{k}, \mathrm{I}}\right)$ and $\widehat{c}_{k, I}=\operatorname{sgn}\left(L_{\mathrm{k}, \mathrm{I}}\right)$, etc. It should be noted that the demapping equations should be similar to those for the SC case $\left(\widetilde{S}_{k, I}^{(i)}\right.$ instead of $\widetilde{s}_{n, I}^{(i)}$, etc), but the constant parameter $\widehat{\sigma}_{e q}^{2}$ has to be replaced by a subchannel-dependent parameter: $\widehat{\sigma}_{k}^{2}(m)=$ $\frac{\widehat{\sigma}_{N}^{2}}{\beta^{2} \sum_{q=1}^{Q}\left|\hat{H}_{k}^{(q)}\right|^{2}}$, where $\widehat{\sigma}_{N}^{2}$ is the estimated variance of $N_{k}^{(q)}$ $\left(k^{t h}\right.$ component of the frequency-domain, input noise vector $\left.\mathbf{N}^{(q)}\right)$.

\section{REFERENCES}

[1] H. Sari, G. Karam, and I. Jeanclaude, "An Analysis of Orthogonal Frequency-division Multiplexing for Mobile Radio Applications," Proc. IEEE VTC'94, Jun. 1994.

[2] A. Gusmão, R. Dinis, J. Conceição, and N. Esteves, "Comparison of Two Modulation Choices for Broadband Wireless Communications," IEEE VTC'2000, May 2000.

[3] D. Falconer, S. Ariyavisitakul, A. Benyamin-Seeyar, and B. Eidson, "Frequency Domain Equalization for Single-Carrier Broadband Wireless Systems," IEEE Comm. Mag., vol. 4, no. 4, Apr. 2002.

[4] R. Dinis and A. Gusmão, "A Class of Nonlinear Signal Processing Schemes for Bandwidth-Efficient OFDM Transmission with Low Envelope Fluctuation," IEEE Trans. on Comm., vol. 52, Nov. 2004.

[5] J. Tellado, L. Hoo, and J. Cioffi, "Maximum Likelihood Detection of Nonlinearly Distorted Multicarrier Symbols by Iterative Decoding," IEEE Trans. on Comm., vol. 51, Feb. 2003.

[6] A. Gusmão, R. Dinis, and P. Torres, "Low-PMEPR OFDM Transmission with an Iterative Receiver Technique for Cancellation of Nonlinear Distortion," IEEE VTC'05 (Fall), vol. 4, Sep. 2005.

[7] M. Tüchler and J.Hagenauer, "Linear Time and Frequency Domain Turbo Equalization," IEEE VTC'01, May 2001.

[8] A. Gusmão, P. Torres, R. Dinis, and N. Esteves, "A Turbo FDE Technique for Reduced-CP SC-Based Block Transmission Systems," IEEE Trans. on Comm., vol. 55, no. 1, Jan. 2007.

[9] P. Torres and A. Gusmão, "Iterative Receiver Technique for Reduced CP Reduced PMEPR OFDM Transmission," IEEE VTC'07 (Spring), Apr. 2007.

[10] , "Evaluation of Clipped-OFDM and SC/FDE Alternatives for Block Transmission Using Iterative Receiver Techniques," IEEE ICC'07, Jun. 2007.

[11] G. Caire, G. Taricco, and E. Biglieri, "Bit-interleaved Coded Modulation," IEEE Transactions on Communications'98, vol. 44, no. 3, May 1998.

[12] K. Bruninghaus and H. Rohling, "Multi-carrier Spread Spectrum and its Relationship to Single-carrier Transmission," IEEE Vehicular Technology Conference, VTC'98 (Spring), vol. 3, no. 3, May 1998.

[13] A. Viterbi, "An Intuitive Justification and a Simplified Implementation of the MAP Decoder for Convolutional Codes," IEEE Journal on Selected Areas on Communications, JSAC'98, vol. 16, no. 2, pp. 260-264, Feb. 1998. 